\title{
Análise Estatística de Notas e Interações em Cursos a Distância
}

\author{
Enyo Gonçalves $^{1,2}$, Jéssyka Vilela ${ }^{1,2}$, Jeandro Bezerra ${ }^{1,2}$ \\ ${ }^{1}$ Universidade Federal do Ceará - Campus de Quixadá \\ ${ }^{2}$ Centro de Informática - Universidade Federal de Pernambuco (UFPE), Recife, Brasil \\ $\{e j t g, j f f v, j m b\} c i n . @ u f p e . b r$
}

\begin{abstract}
Courses offered in distance education require dedication, commitment and discipline from students. The interactions of the students in virtual environments of education can be an indicator of their learning. This work investigates the interactions and grades among students and their correlation, besides interactions of tutors in a virtual learning environment. Statistical analysis of interactions and grades in three courses were performed. We identified a correlation between interactions and notes of the students, a tool was built and made available to the community. The data can be used to better understand the courses in order to support the pedagogical decision making and the tool can be used by other courses.
\end{abstract}

Resumo. Cursos ofertados na modalidade de Educação a Distância (EAD) requerem dedicação, comprometimento e disciplina dos alunos. As interações dos alunos em ambientes virtuais de aprendizagem podem ser um indicador do seu aprendizado. Este trabalho investiga as notas e interações de alunos e sua correlação, além de interações de tutores em um Ambiente Virtual de Aprendizagem. Análises estatísticas de interações e notas em três cursos EAD foram realizadas. Nós identificamos correlação entre interações e notas dos alunos e uma ferramenta foi construída e disponibilizada para a comunidade. Os dados podem ser utilizados para uma melhor compreensão dos cursos de modo a auxiliar a tomada de decisão pedagógica e a ferramenta pode ser utilizada no contexto de outros cursos a distância.

\section{Introdução}

A Educação a Distância (EAD) é uma modalidade de ensino que é uma realidade no contexto da educação brasileira. Uma variedade de ofertas de cursos pode ser identificada, seja no nível de cursos de capacitação, técnicos, graduação e pós-graduação. Um dos casos de destaque trata-se da Universidade Aberta do Brasil (UAB) ${ }^{1}$, a qual oferece cursos de graduação e pós-graduação de forma semipresencial em parceria com as universidades de cada estado.

A UAB em parceria com a Universidade Estadual do Ceará (UECE) oferece vários cursos de graduação e pós-graduação nessa modalidade. Um dos principais diferenciais desses cursos é a grande quantidade de dados gerada pelas interações no Ambiente Virtual

\footnotetext{
${ }^{1}$ http://www.capes.gov.br/uab
} 
VII Congresso Brasileiro de Informática na Educação (CBIE 2018)

Anais do XXIX Simpósio Brasileiro de Informática na Educação (SBIE 2018)

de Aprendizagem (AVA), o que permite novas possibilidades para estudar e compreender estas interações [Detoni et al. 2014].

Um dos grandes desafios neste modelo de educação trata-se do acompanhamento dos alunos. Portanto, ferramentas que viabilizem um acompanhamento mais efetivo podem contribuir de maneira significativa neste sentido. Dois indicadores interessantes relacionados ao progresso dos alunos são as notas e a participação nas atividades, fóruns e outros recursos de interação do AVA como o Moodle.

Um papel de destaque no AVA é o do tutor [dos Santos and Falcão 2017]. Este é responsável por acompanhar os alunos seja pela frequência nas discussões, entrega de atividades bem como para tirar dúvidas. Essas interações entre alunos e tutores no AVA podem ser utilizadas para aferir o comprometimento e progresso dos alunos. Além disto, o grau dessas interações pode ter correlação com as notas dos alunos.

Sendo assim, este trabalho tem como objetivo investigar as interações dos alunos no AVA por meio de uma análise estatística comparativa entre dados de cursos EAD da UAB em parceria com a UECE. Esta análise envolve: i) apresentar os dados e comparar as interações dos alunos de cursos (Licenciatura em Computação, Pedagogia e Ciências Biológicas) pertencentes a três áreas de conhecimento distintas (Exatas, Humanas e Ciências Biológicas); ii) investigar a correlação entre a quantidade de interações do aluno e a média geral da nota; iii) analisar as interações dos alunos com média geral boa $\mathrm{x}$ alunos com média geral ruim e iv) analisar as interações dos tutores nestes cursos. Finalmente, foi desenvolvido uma ferramenta que pode ser utilizada por outros pesquisadores que utilizem o Moodle como AVA.

Os resultados desta investigação são importantes para que a equipe pedagógica da UAB/UECE possa ter mais subsídios para fazer o acompanhamento dos cursos. Sendo assim, pode-se planejar as atividades a serem desenvolvidas com os alunos bem como acompanhar a aprendizagem e a interatividade com os tutores. Outra contribuição é a possibilidade de acompanhar a relação entre interações e médias dos alunos e o progresso destas duas variáveis.

O artigo está organizado da seguinte forma: a fundamentação teórica e trabalhos relacionados são discutidos na Seção 2. A metodologia é descrita na Seção 3. Os resultados, discussões das análises estatísticas bem como a ferramenta criada são apresentados na Seção 4. Finalmente, as conclusões e trabalhos futuros são explorados na Seção 5.

\section{Fundamentação teórica e Trabalhos Relacionados}

Nesta seção, são discutidos aspectos da EAD no Brasil bem como trabalhos relacionados.

\subsection{Educação a Distância no Brasil}

Na modalidade EAD, são utilizados materiais auto didáticos, produzidos especificamente para esta finalidade e distribuídos utilizando diversos meios de comunicação [Bordevane D 1988].

De acordo com o [Portal Do Ministério Da Educação 2018], em 1996, a EAD surge oficialmente no Brasil, sendo as bases legais para essa modalidade de educação, estabelecidas pela Lei de Diretrizes e Bases (LDB) da Educação Nacional n. 9.394.Entretanto, a EAD somente foi regulamentada em 2005 pelo Decreto 
VII Congresso Brasileiro de Informática na Educação (CBIE 2018)

Anais do XXIX Simpósio Brasileiro de Informática na Educação (SBIE 2018)

n. 5.622, com normatização definida na Portaria Ministerial n. 4.361 de 2004 [Portal Do Ministério Da Educação 2018].

A partir de 1996, com a reforma da LDB, a EAD teve seu primeiro apoio legal, permitindo aproximação em todos os níveis educacionais. Apesar da regulamentação permitir utilizar a EAD em todos contextos educacionais, é exatamente no Ensino Superior que ela vem tendo grande destaque. Atualmente, é grande o número de instituições que ofertam cursos superiores na modalidade EAD no Brasil. Este é um reflexo da popularização desta modalidade de ensino no país.

Segundo [Silva 2003], a interatividade é característica fundamental para a educação on-line ao permitir redimensionar o conceito de distância e contribuir para aprendizagem colaborativa. Portanto, os sujeitos envolvidos no processo aprendem não só com o material didático como também através da atividade dialógica entre eles. Assim sendo, os AVAs exercem papel fundamental neste contexto.

\subsection{Ambientes Virtuais de Aprendizagem em Educação a Distância}

Segundo [Faria 2003], é possível estabelecer facilmente a comunicação entre os sujeitos envolvidos na EAD, uma vez que a interação é oportunizada pelas Tecnologias de Informação e Comunicação (TICs) disponíveis, como, por exemplo, os AVAs.

No cenário atual, os AVAs são as ferramentas que viabilizam a realização de cursos nesta modalidade. De modo geral, um AVA refere-se ao uso de recursos digitais de comunicação, principalmente, por meio de softwares educacionais via web que reúnem diversas ferramentas de interação [Valentini and Soares 2005].

Os AVAs podem ser considerados aliados da teoria sócio interacionista, uma vez que, por meio deles, oportunizam-se trocas entre os indivíduos e o contato com o objeto de conhecimento. Nesses ambientes, o professor acompanha e media a construção do conhecimento de seus alunos. Parte-se da interação para estabelecer a internalização, caracterizada por [Vigotski 1999] como a "reconstrução interna de uma operação externa".

Vários AVAs vêm sendo disponibilizados para uso, dentre eles pode-se citar o Moodle (Modular Object-Oriented Dynamic Learning Environment) por ser amplamente utilizado em cursos de EAD nacional e internacionalmente. O Moodle trata-se de um software livre disponível para download ${ }^{2}$ que pode ser utilizado sem custos e vem viabilizando o desenvolvimento de cursos na modalidade EAD ao redor do mundo. Este software é disponibilizado em uma versão padrão (genérica) que pode ser customizada para o uso em determinado curso/instituição de ensino.

\subsection{Trabalhos Relacionados}

Existem diversos trabalhos no contexto de análise de interações e notas em cursos a distância, um deles é o de [Detoni et al. 2014] no qual foram utilizados modelos de aprendizagem de máquinas para predição de reprovação. Porém, os trabalhos investigados durante esta pesquisa não enfatizam uma abordagem estatística experimental.

Fatores que afetam o desempenho de estudantes iniciantes em um curso EAD são analisados no trabalho de [Ramos et al. 2014]. Por meio da aplicação de questionários o

\footnotetext{
${ }^{2}$ https://www.moodle.org
} 
VII Congresso Brasileiro de Informática na Educação (CBIE 2018)

Anais do XXIX Simpósio Brasileiro de Informática na Educação (SBIE 2018)

cruzamento de algumas variáveis com o desempenho dos alunos, os resultados apontaram que a experiência prévia com EAD, conhecimento satisfatório em informática e facilidade de adaptação a novas tecnologias tiveram relação significativa com o desempenho do aluno na primeira disciplina do curso.

Uma ferramenta é proposta no trabalho de [dos Santos and Falcão 2017] para mitigar problemas enfrentados por tutores em AVAs. Esta usa algoritmos de classificação e agrupamento para fornecer uma visão aprimorada do desempenho e comportamento dos alunos na plataforma.

Uma análise qualitativa das contribuições textuais registradas por alunos em fóruns é discutida no trabalho de [Azevedo et al. 2011]. A partir da identificação da relevância das mensagens, é possível ter subsídios para avaliar os discentes que necessitam de maior auxílio.

O padrão de comportamento de alunos e professores de cursos EAD da Universidade Federal do Ceará é descrito em [Pequeno et al. 2014]. As interações virtuais nos fóruns segundo duas perspectivas de oferta de disciplinas são apresentadas.

Este trabalho complementa o estado da arte ao demonstrar a correlação entre a média dos alunos em AVAs e a interação dos tutores em cursos de diferentes áreas (exatas, humanas, e da saúde).

\section{Metodologia}

O objetivo principal deste trabalho consiste na análise da correlação entre as interações dos alunos e tutores no AVA bem como as médias das notas. A primeira etapa consistiu na realização dos testes de normalidade de Kolmogorov-Smirnov e Shapiro-Wilk (Seção 4.2) das variáveis listadas na Tabela 1.

Em virtude da rejeição da normalidade dos dados, nas análises foram utilizados testes estatísticos não-paramétricos. A correlação entre interações e médias das notas dos alunos (Seção 4.3) foi determinada utilizando o teste de correlação de postos de Spearman. O teste de wilcoxon para amostras independentes foi aplicado para analisar a quantidade média de interações dos alunos e suas notas (Seção 4.4). Esta seção também investiga o comportamento das interações dos tutores dos três cursos por meio do teste de Kruskal-Wallis. A Tabela 4 apresenta as hipóteses estatísticas bem como os testes de hipóteses aplicados.

\subsection{Especificação da Amostra}

A UAB/UECE possui dez cursos de Graduação, três de Especialização e um de Mestrado. Estes cursos são distribuídos em 17 polos no estado do Ceará com um corpo discente de mais de 2500 alunos. As amostras foram retiradas do banco de dados em produção (Postgres) que contém as interações dos alunos e tutores de todos os polos, a partir do ano de 2010.

\subsection{Variáveis e Hipóteses}

As variáveis envolvidas neste trabalho são apresentadas na Tabela 1. As três amostras de alunos analisadas nesse trabalho são descritas na Tabela 2. A Tabela 3.2 apresenta a estatística descritiva dos dados das interações dos tutores dos três cursos. 
VII Congresso Brasileiro de Informática na Educação (CBIE 2018)

Anais do XXIX Simpósio Brasileiro de Informática na Educação (SBIE 2018)

\begin{tabular}{|c|c|}
\hline NI = Quantidade de Interações do Aluno no AVA & C = Curso do aluno \\
\hline NT = Quantidade de Interações do Tutor no AVA & $\mathrm{N}=$ Tamanho de cada amostra \\
\hline MF = Média da Nota do aluno & \\
\hline
\end{tabular}

Tabela 1. Variáveis investigadas.

\begin{tabular}{|c|c|c|c|c|}
\hline Cursos & Qtde de Alunos & Polos & Média de Interações & Média das Notas \\
\hline Licenciatura em Computação & 338 & 7 & 125,3 & 42,53 \\
\hline Pedagogia & 463 & 9 & 47,41 & 69,68 \\
\hline C. Biológicas & 284 & 5 & 297,90 & 51,35 \\
\hline
\end{tabular}

Tabela 2. Dados amostrais alunos.

\begin{tabular}{|c|c|c|c|c|c|c|}
\hline Curso & Qtde de Tutores & Média & Mediana & Moda & Mínimo & Máximo \\
\hline Licenciatura em Computação & 21 & 338,1 & 264 & 1 & 1 & 1026 \\
\hline Pegagogia & 23 & 125,8 & 105 & 35 & 2 & 491 \\
\hline C. Biológicas & 31 & 280,8 & 198 & 1 & 1 & 1307 \\
\hline
\end{tabular}

Tabela 3. Dados amostrais e análise exploratória das interações dos tutores.

\begin{tabular}{|l|c|l|}
\hline \multirow{2}{*}{ Hipóteses para teste de Spearman } & $\mathrm{H}_{0}$ & $\begin{array}{l}\text { Não existe relação linear entre as interações e } \\
\text { e as médias das notas dos alunos }\end{array}$ \\
\cline { 2 - 3 } & $\mathrm{H}_{1}$ & $\begin{array}{l}\text { Existe relação linear entre as interações } \\
\text { e as médias das notas dos alunos }\end{array}$ \\
\hline \multirow{2}{*}{ Hipóteses para o teste de Wilcoxon } & $\mathrm{H}_{0}$ & $\begin{array}{l}\text { A mediana das interações dos alunos } \\
\text { com notas }>=7 \text { é igual a mediana } \\
\text { das interações dos alunos com notas }<7\end{array}$ \\
\cline { 2 - 3 } & $\mathrm{H}_{1}$ & $\begin{array}{l}\text { A mediana das interações dos alunos com notas }>=7 \text { é maior que a mediana } \\
\text { das interações dos alunos com notas }<7\end{array}$ \\
\hline \multirow{2}{*}{ Hipótese para teste de Kruskal-Wallis } & $\mathrm{H}_{0}$ & A quantidade de interações dos tutores dos três cursos são iguais. \\
\cline { 2 - 3 } $\begin{array}{l}\text { Hipóteses para o teste de Wilcoxon } \\
\text { (comparação } 3 \text { amostras dois a dois) }\end{array}$ & $\mathrm{H}_{1}$ & A quantidade de interações dos tutores em pelo menos um dos três cursos é diferente. \\
\cline { 2 - 3 } & $\mathrm{H}_{0}$ & A quantidade de interações dos tutores nos dois cursos são iguais. \\
\hline
\end{tabular}

Tabela 4. Testes de Hipóteses dos três cursos.

\section{Resultados}

Um aspecto importante ao realizar análises estatísticas é organizar e resumir os dados utilizando maneiras que facilitem sua interpretação e análise subsequente. A representação visual dos dados amostrais, através de técnicas gráficas como o histograma e o box-plot, permite investigar a simetria e estatísticas dos dados como média, moda e mediana.

\subsection{Estatística Descritiva}

A Figura 1 apresenta o histograma da quantidade de interações no AVA dos alunos dos três cursos analisados nesse trabalho. A disposição gráfica dos três histogramas indica que a variável interações não segue uma distribuição normal em nenhum dos cursos.

Os histogramas da quantidade de interações dos alunos dos cursos de computação e biologia apresentam uma assimetria à direita uma vez que a média é maior que a mediana. Apesar do histograma referente as interações do curso de pedagogia apresentar uma concentração dos dados a esquerda menor, os dados das interações nesse curso também são assimétricos à direita.

Os histogramas das médias das notas dos alunos dos três cursos também são exibidos na Figura 1. A análise desses gráficos permite concluir que os dados das médias 
VII Congresso Brasileiro de Informática na Educação (CBIE 2018)

Anais do XXIX Simpósio Brasileiro de Informática na Educação (SBIE 2018)
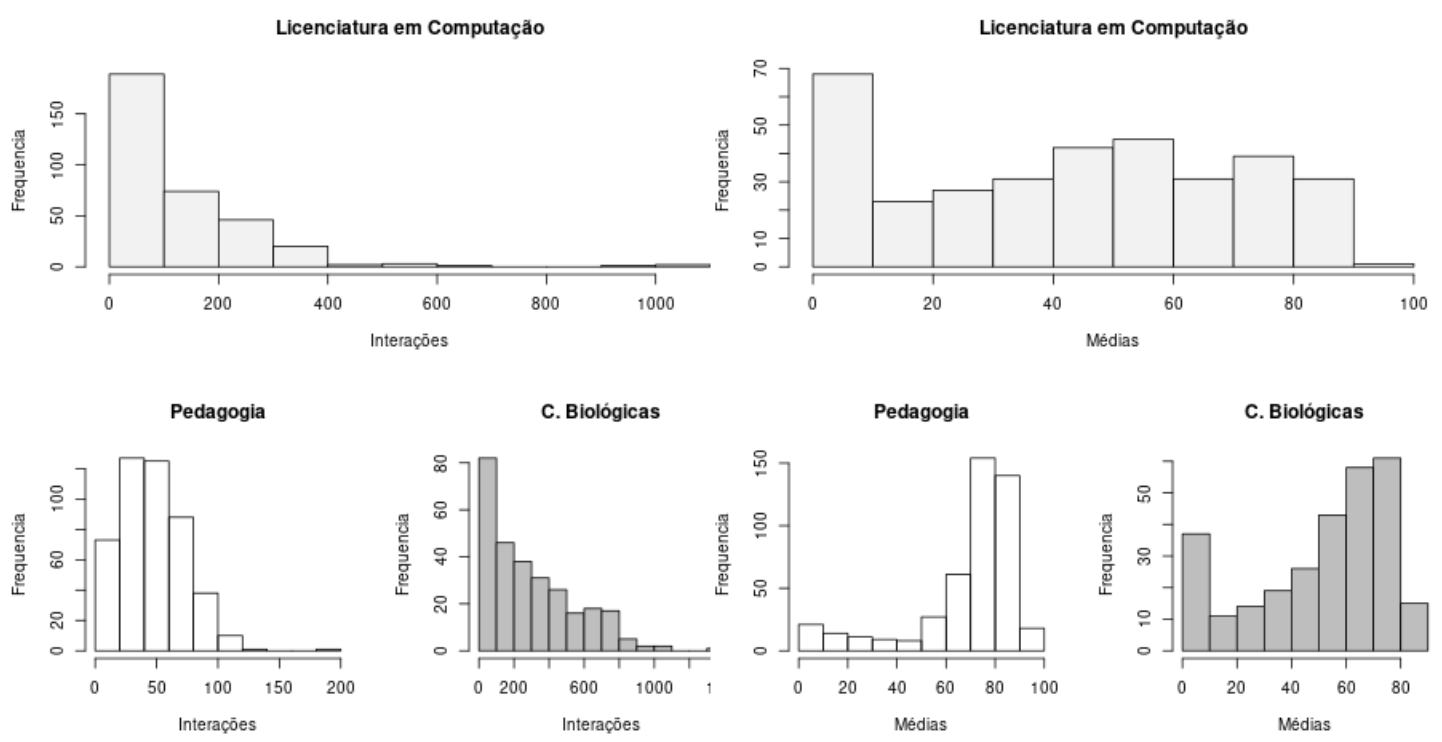

Figura 1. Histogramas da Quantidade de Interações e Médias das notas dos três cursos.

das notas são assimétricos à esquerda uma vez que as médias dos cursos de computação, pedagogia e biologia são menores que as medianas.

A Figura 2 apresenta os boxplots da variável interações dos alunos dos três cursos complementando visualmente os histogramas apresentados anteriormente. O curso de computação é o que apresenta a maior quantidade de outliers seguido pelo curso de biologia e, por fim, pedagogia. A figura apresenta também os boxplots da variável médias das notas dos alunos dos três cursos. O curso de pedagogia possui vários outliers enquanto que computação e pedagogia não apresentam outliers.
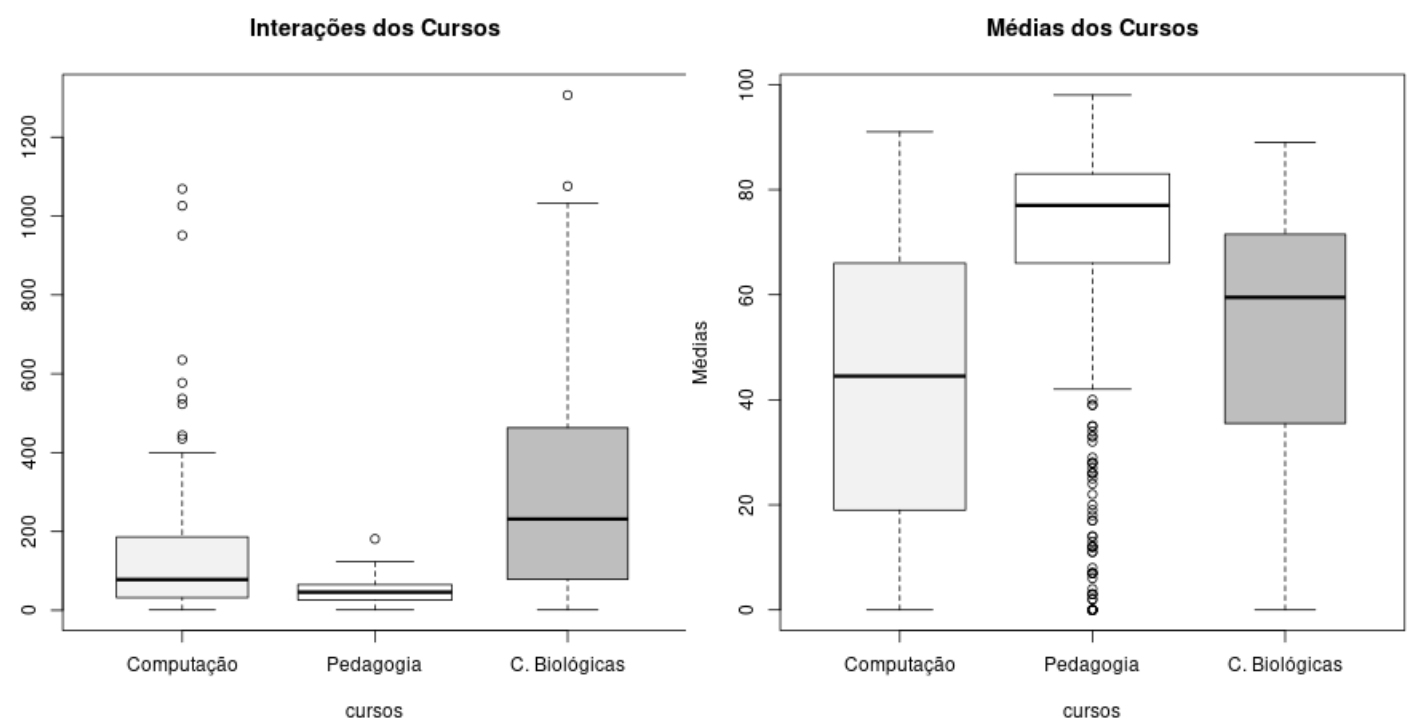

Figura 2. Boxplots das interações e das médias das notas dos três cursos. 
VII Congresso Brasileiro de Informática na Educação (CBIE 2018)

Anais do XXIX Simpósio Brasileiro de Informática na Educação (SBIE 2018)

A Tabela 4.1 apresenta o resumo numérico (média, mediana, moda, valor mínimo e valor máximo) dos dados das interações e notas para os três cursos.

\begin{tabular}{|c|c|c|c|c|c|c|}
\hline Curso & variável & Média & Mediana & Moda & Valor Mínimo & Valor Máximo \\
\hline \multirow{2}{*}{ Computação } & Interações & 125,3 & 78 & 2 & 1 & 1069 \\
& Média & 42,53 & 44,5 & 0 & 0 & 91 \\
\hline \multirow{2}{*}{ Pegagogia } & Interações & 47,41 & 46 & 25 & 1 & 181 \\
& Média & 69,68 & 77 & 80 & 0 & 98 \\
\hline \multirow{2}{*}{ C. Biológicas } & Interações & 297,9 & 231,5 & 8 & 1 & 1387 \\
& Média & 51,35 & 59,5 & 0 & 0 & 89 \\
\hline
\end{tabular}

Tabela 5. Análise exploratória das interações dos alunos e das notas.

\subsection{Teste de Normalidade}

A característica heterogênea das variáveis de interações e médias das notas dos alunos dos três cursos motivou a realização dos testes estatísticos de normalidade dos dados. Neste trabalho, foram utilizados os testes de normalidade de Kolmogorov-Smirnov e ShapiroWilk cujos resultados são listados nas Tabelas 6, 7 e 8.

\begin{tabular}{|c|c|c|c|}
\hline Curso & Teste & Estatística & $\mathrm{p}$-value \\
\hline \multirow{2}{*}{ Computação } & Kolmogorov-Smirnov & $\mathrm{D}=0,18905$ & $6,438 \mathrm{e}-11$ \\
\cline { 2 - 4 } & Shapiro-Wilk & $\mathrm{W}=0,74417$ & $<2,2 e-16$ \\
\hline \multirow{2}{*}{ Pedagogia } & Kolmogorov-Smirnov & $\mathrm{D}=0,0537$ & 0,1384 \\
\cline { 2 - 4 } & Shapiro-Wilk & $\mathrm{W}=0,97519$ & $4,389 e-07$ \\
\hline \multirow{2}{*}{ C. Biológicas } & Kolmogorov-Smirnov & $\mathrm{D}=0,12613$ & 0,000238 \\
\cline { 2 - 4 } & Shapiro-Wilk & $\mathrm{W}=0,90638$ & $2,724 \mathrm{e}-12$ \\
\hline
\end{tabular}

Tabela 6. Testes de Normalidade para interações dos alunos dos três cursos.

\begin{tabular}{|c|c|c|c|}
\hline Curso & Teste & Estatística & p-value \\
\hline \multirow{2}{*}{ Computação } & Kolmogorov-Smirnov & $\mathrm{D}=0,082839$ & 0,01934 \\
\cline { 2 - 4 } & Shapiro-Wilk & $\mathrm{W}=0,93869$ & $1,337 \mathrm{e}-10$ \\
\hline \multirow{2}{*}{ Pedagogia } & Kolmogorov-Smirnov & $\mathrm{D}=0,20853$ & $<2,2 e-16$ \\
\cline { 2 - 4 } & Shapiro-Wilk & $\mathrm{W}=0,77315$ & $<2,2 e-16$ \\
\hline \multirow{2}{*}{ C. Biológicas } & Kolmogorov-Smirnov & $\mathrm{D}=0,13534$ & $6,064 \mathrm{e}-05$ \\
\cline { 2 - 4 } & Shapiro-Wilk & $\mathrm{W}=0,89156$ & $2,284 \mathrm{e}-13$ \\
\hline
\end{tabular}

Tabela 7. Testes de Normalidade para médias das notas dos alunos dos três cursos.

Portanto, é possível concluir, a uma confiança de 95\%, que existe evidências suficientes para rejeitar as hipóteses de que as variáveis interações dos alunos e tutores e médias das notas dos alunos dos três cursos provêm de populações normalmente distribuídas.

Os dados das interações dos três cursos investigados neste trabalho indicam que os alunos do curso de Ciências Biológicas interagem mais com o AVA seguidos pelos alunos de Licenciatura em Computação e por Pedagogia. Este resultado é interessante uma vez que se esperava que os alunos de computação interagissem mais no AVA, uma vez que possuem, de forma geral, maior familiaridade com tecnologias. 
VII Congresso Brasileiro de Informática na Educação (CBIE 2018)

Anais do XXIX Simpósio Brasileiro de Informática na Educação (SBIE 2018)

\begin{tabular}{|c|c|c|c|}
\hline Curso & Teste & Estatística & p-value \\
\hline \multirow{2}{*}{ Computação } & Kolmogorov-Smirnov & $\mathrm{D}=0,13441$ & 0,7951 \\
\cline { 2 - 4 } & Shapiro-Wilk & $\mathrm{W}=0,90736$ & 0,04872 \\
\hline \multirow{2}{*}{ Pedagogia } & Kolmogorov-Smirnov & $\mathrm{D}=0,18802$ & 0,3904 \\
\cline { 2 - 4 } & Shapiro-Wilk & $\mathrm{W}=0,79405$ & 0,0003082 \\
\hline \multirow{2}{*}{ C. Biológicas } & Kolmogorov-Smirnov & $\mathrm{D}=0,18173$ & 0,2575 \\
\cline { 2 - 4 } & Shapiro-Wilk & $\mathrm{W}=0,84079$ & 0,0003244 \\
\hline
\end{tabular}

Tabela 8. Testes de Normalidade para Interações dos Tutores dos Três Cursos.

Apesar da elevada quantidade das interações dos alunos de biologia, essa característica não é observada nos tutores desse curso. Os tutores de Licenciatura em Computação são os que mais interagem no AVA seguidos pelos tutores do curso de Ciências Biológicas. Por outro lado, a quantidade reduzida de interações do curso de pedagogia também é observada nos tutores.

Em relação às médias das notas verificou-se que os alunos de Pedagogia possuem a maior média das notas seguidos pelos alunos de Ciências Biológicas e Licenciatura em Computação. Este era um resultado de certa forma esperado, em virtude dos cursos da área de exatas possuírem altas taxas de evasão.

\subsection{Análise de Correlação entre Interações e Médias das Notas dos Alunos}

O resultado do teste de correlação de postos de Spearman para as interações e médias das notas dos alunos dos três cursos é apresentado na Tabela 9. É possível concluir, a uma confiança de $95 \%$, que existe evidências suficientes para rejeitar a hipótese de que não existe correlação linear entre as variáveis interações e médias das notas para os três cursos investigados.

\begin{tabular}{|c|c|c|}
\hline \multirow{2}{*}{ Curso } & \multicolumn{2}{|c|}{ Spearman } \\
& Coeficiente de correlação & p-value \\
\hline Computação & 0,661268 & $<2.2 e-16$ \\
\hline Pedagogia & 0,408531 & $<2.2 e-16$ \\
\hline C. Biológicas & 0,6719453 & $<2.2 e-16$ \\
\hline
\end{tabular}

Tabela 9. Resultado do teste de correlação de postos de Spearman para as interações e médias das notas dos alunos dos três cursos.

Os testes de correlação indicam a existência de uma correlação linear positiva entre as interações e as médias das notas dos alunos nos cursos de Licenciatura em Computação e de C. Biológicas. Por outro lado, a correlação é positivamente moderada no curso de Pedagogia.

\subsection{Testes de Hipótese}

Os resultados do teste de wilcoxon permitem rejeitar a hipótese de que as interações dos dois grupos (média da nota $>=7$ e média da nota $<7$ ) têm valores de medianas iguais em todos os três cursos estudados. Portanto, existe evidência a nível de confiança de $95 \%$ para afirmar que a mediana das interações do grupo de alunos com notas $>=7$ é maior que a mediana das interações do grupo de alunos com notas $<7$ em todos os três cursos analisados. 
VII Congresso Brasileiro de Informática na Educação (CBIE 2018)

Anais do XXIX Simpósio Brasileiro de Informática na Educação (SBIE 2018)

\begin{tabular}{|c|c|c|}
\hline \multirow{2}{*}{ Curso } & \multicolumn{2}{|c|}{ Wilcoxon } \\
& Estatística do Teste & $\mathrm{p}$-value \\
\hline Computação & $\mathrm{W}=16098$ & $<2.2 e-16$ \\
\hline Pedagogia & $\mathrm{W}=33025$ & $<2.2 e-16$ \\
\hline C. Biológicas & $\mathrm{W}=13816$ & $<2.2 e-16$ \\
\hline
\end{tabular}

Tabela 10. Resultado do teste de wilcoxon para as interações dos alunos e médias das notas dos três cursos.

Outro aspecto analisado neste trabalho foi o comportamento das interações dos tutores dos três cursos no AVA. Os resultados do Teste de Kruskal-Wallis (Tabela 11) permitem concluir, a uma confiança de $95 \%$, que não existe evidências suficientes para rejeitar a hipótese de que as quantidades de interações dos tutores dos três cursos são estatisticamente iguais.

Dessa forma, os cursos foram analisados aos pares por meio do Teste de Wilcoxon cujos resultados também são listados na Tabela 11. Esses resultados indicam que há diferença significativa entre as interações dos tutores dos seguintes pares de cursos: $\mathrm{C}$. Biológicas e Licenciatura em Computação bem como no par C. Biológicas e Pedagogia. Por outro lado, a hipótese de igualdade das interações dos tutores dos cursos de Pedagogia e Licenciatura em Computação foi rejeitada. Portanto, não é possível realizar inferências a partir desse teste, sendo necessário aumentar o tamanho da amostra. É importante observar que os testes para mais de duas amostras tendem a ser um pouco menos precisos do que os de apenas duas amostras e apesar da hipótese nula não ter sido rejeitada, o p-value resultante desse teste é próximo do nível de significância adotado (5\%).

\begin{tabular}{|c|c|c|}
\hline \multicolumn{3}{|c|}{ Kruskal-Wallis chi-squared $=5,0583, \mathrm{df}=2, \mathrm{p}$-value $=0,07973$} \\
\hline \multicolumn{3}{|c|}{ Wilcoxon } \\
\hline Curso & Computação & Pedagogia \\
\hline Pedagogia & $\mathrm{W}=335,5$ e $\mathrm{p}$-value $=0,02801$ & - \\
\hline Biologia & $\mathrm{W}=377$ e $\mathrm{p}$-value $=0,3415$ & $\mathrm{~W}=439,5$ e $\mathrm{p}$-value $=0,1489$ \\
\hline
\end{tabular}

Tabela 11. Resultado do teste de Kruskal-Walllis e Wilcoxon para a quantidade de interações dos tutores nos três cursos.

Durante a análise dos dados, foi desenvolvido a ferramenta estatística denominada Interactions and Scores Analysis Tool (ISAT) que pode ser utilizada para fazer análises semelhantes às apresentadas neste trabalho. A ferramenta está acoplada ao MOODLE e é capaz de fazer as análises estatísticas apresentada neste artigo. Dessa forma, ela facilita futuras análises para a equipe pedagógica de cursos de EAD que utilizem o Moodle como AVA. A ferramenta está disponível para download em www.cin.ufpe.br/ ejtg/ ISAT.

\section{Conclusão e Trabalhos Futuros}

Este artigo apresentou uma análise dos dados de notas de alunos e interações de alunos e tutores dos cursos da UAB/UECE. Foram analisados os dados dos cursos de Ciências Biológicas, Licenciatura em Computação e Pedagogia em relação às interações e notas dos alunos. A análise de interações apresentou uma surpresa, uma vez que os alunos do 
VII Congresso Brasileiro de Informática na Educação (CBIE 2018)

Anais do XXIX Simpósio Brasileiro de Informática na Educação (SBIE 2018)

curso de Ciências Biológicas interagem mais que os alunos do curso de Licenciatura em Informática. Identificamos correlação entre médias dos alunos e suas interações e que os alunos com os alunos com notas maiores que 7 interagem mais que alunos com notas menores que 7. Também analisamos as interações dos tutores entre os três cursos e conseguimos identificar que os tutores de Licenciatura em Computação e Ciências Biológicas interagem mais que os tutores do curso de Pedagogia.

Os resultados apresentados foram entregues a equipe pedagógica responsável para uma melhor análise sobre as interações e notas dos alunos e interações dos tutores. Além disto, a ferramenta disponibilizada pode ser útil não somente para os cursos analisados, mas também para outros cursos a distância que utilizem o Moodle como AVA.

Como trabalhos futuros, pretende-se realizar um comparativo entre os demais cursos da UAB/UECE em relação aos critérios de análise empregados neste trabalho.

Agradecimentos: Os autores agradecem ao apoio do Conselho Nacional de Desenvolvimento Científico e Tecnológico, Fundação Cearense de Apoio ao Desenvolvimento Científico e Tecnológico e Fundação de Amparo a Ciência e Tecnologia de Pernambuco.

\section{Referências}

Azevedo, B. F. T., Behar, P. A., and Reategui, E. B. (2011). Análise das mensagens de fóruns de discussão através de um software para mineração de textos. In Simpósio Brasileiro de Informática na Educação-SBIE, volume 1.

Bordevane D, J. L. (1988). Pode a educação a distancia ajudar a resolver os problemas educacionais no brasil. Rev. Tecnol. Educ, 17(80/81):31-36.

Detoni, D., Araujo, R. M., and Cechinel, C. (2014). Predição de reprovação de alunos de educação a distância utilizando contagem de interações. In Anais do Simpósio Brasileiro de Informática na Educação, volume 25, pages 896-905.

dos Santos, D. C. V.-B. and Falcão, T. P. (2017). Acompanhamento de alunos em ambientes virtuais de aprendizagem baseado em sistemas tutores inteligentes. In Simpósio Brasileiro de Informática na Educação-SBIE, volume 28, page 1267.

Faria, E. T. (2003). Interatividade e mediação pedagógica na educação a distância.

Pequeno, H., de Ávila, R. L., Cruz, E., Alexandre, M., de Lima, E. T., and de Castro, M. F. (2014). Uma análise de interação em fóruns de ead. In Simpósio Brasileiro de Informática na Educação-SBIE, volume 25, page 1203.

Portal Do Ministério Da Educação (2018). Regulamentação da ead no brasil.

Ramos, J. L. C., Rodrigues, R. L., Silva, J. C. S., and Gomes, A. S. (2014). Analisando fatores que afetam o desempenho de estudantes iniciantes em um curso a distância. In Simpósio Brasileiro de Informática na Educação-SBIE, volume 25, page 99.

Silva, M. (2003). Educação online: teorias, práticas, legislação, formação corporativa. Edições Loyola.

Valentini, C. and Soares, E. (2005). Fluxos de interação: uma experiência com ambiente de aprendizagem na web. Aprendizagem em ambientes virtuais: compartilhando idéias e construindo cenários. Caxias do Sul: EDUCS, 1:77-86.

Vigotski, L. S. (1999). A formação social da mente. São Paulo. 